\title{
Online DCM Trajectory Adaptation for Push and Stumble Recovery during Humanoid Locomotion
}

\author{
George Mesesan, Johannes Englsberger, Christian Ott
}

\begin{abstract}
In this paper, we present a highly efficient Divergent Component of Motion (DCM) reference trajectory generator capable of adapting online to large perturbations acting on the center-of-mass (push recovery) and on the swing foot (stumble recovery). For push recovery, we propose an analytic solution for a footstep adjustment strategy based on the DCM dynamics. The proposed algorithm considers double support phases explicitly and is active throughout the motion, i.e., during both single and double support phases. For stumble recovery, we introduce a continuous DCM trajectory adaptation based on the instantaneous tracking error of the swing foot. Our method is highly efficient, computing a push recovery solution within 10 microseconds on the robot hardware. Furthermore, it achieves robust locomotion for large external perturbations, which we demonstrate in simulations and experiments with the humanoid robot TORO.
\end{abstract}

\section{INTRODUCTION}

Humanoid robots are expected to be able to interact safely with humans, and work alongside them in cluttered environments [1]. Despite extensive research in the last decades, robust humanoid locomotion remains a challenging problem due to its hybrid dynamics and the constraints on the direction and magnitude of the contact forces. Of particular interest is the ability to maintain balance following strong disturbances, acting either on the center-of-mass (push recovery), or on the swing foot (stumble recovery).

One of the most popular models for humanoid locomotion is the Linear Inverted Pendulum (LIP) [2]. More recently, the Divergent Component of Motion (DCM) [3] was introduced with the goal of simplifying the trajectory generation and control by focusing on the unstable component of the CoM dynamics. Given a sequence of preplanned footsteps, an efficient algorithm for generating reference trajectories using the three-dimensional DCM [4] was introduced in our previous work [5]. A DCM tracking controller [4] was implemented in a passivity-based whole-body torque controller to achieve dynamic walking on compliant and uneven terrain [6].

To improve robustness in the presence of large perturbations, researchers have looked at nature for inspiration. An experimental study on human balancing [7] mentioned three basic strategies for balancing (ankle, hip, and stepping strategies), and has shown that, contrary to traditional views, humans prefer the stepping over the hip strategy for stabilizing after large perturbations. In robotics, several frameworks have been proposed to achieve robust locomotion and disturbance rejection. A model predictive control (MPC)

All authors are with the Institute of Robotics and Mechatronics, German Aerospace Center (DLR), Wessling, Germany. Contact: george.mesesan@dlr.de scheme was introduced by Herdt et. al [8] to compute step locations in reaction to disturbances, using the zero moment point (ZMP) dynamics while minimizing the CoM jerk. Aftab et al. [9] proposed an MPC framework combining the three strategies for stabilizing a LIP model with a flywheel in simulations, while Shafiee-Ashiani et al. [10] combined ankle, hip, and stepping strategies in an MPC framework using the DCM instead of the whole CoM dynamics. Urata et al. [11] selected optimal ZMP-CoM pairs through preview control for online footstep adjustment in response to pushes or sudden changes in direction or speed. Another approach has been to formulate and solve online footstep optimization problems, based either on the LIP model [12], or on an analytic solution of the DCM for general ZMP trajectories [13]. Griffin et al. [14] proposed a quadratic optimization problem combining a proportional feedback controller with a controller that adjusts multiple footsteps, using a recursive algorithm based on DCM dynamics with constant ZMP.

A footstep adaptation algorithm based on DCM dynamics and using an exponential interpolation of the ZMP trajectory was proposed in [15], but the step adaptation was active only during the single support phase. Jeong et al. [16] presented a robust walking controller based on the DCM dynamics using an online optimization solver to combine the ankle, hip, and stepping strategies. Yet, an important missing aspect of their method is the absence of double support phases. In our previous work [5], we presented a push recovery algorithm using a conservative estimate of the DCM error at the end of the single or double support phase.

The main contributions of this work are: (i) We propose a highly efficient push recovery algorithm based on the DCM dynamics with an analytic solution for combining the ankle and stepping strategies. Unlike existing works, our algorithm considers double support phases explicitly, and is continuously active during both single and double support phases. (ii) We introduce a stumble recovery algorithm as a continuous DCM reference trajectory adaptation based on the instantaneous tracking error of the swing foot.

The paper is organized as follows: Section II reviews the main results regarding DCM from our previous work [4], [5], [17], and introduces the notation that is used throughout the paper. Section III gives an overview of the reference trajectory generator, with Section IV detailing the push recovery algorithm, and Section $\mathrm{V}$ presenting the stumble recovery approach. Section VI describes the performed simulations and experiments that demonstrate the robustness of the overall approach. Section VII presents the conclusions and gives an outline for future work. 


\section{Fundamentals (PREViOUS WORK)}

The three-dimensional Divergent Component of Motion (DCM) $\boldsymbol{\xi}$ was defined in [4] as $\boldsymbol{\xi}:=\boldsymbol{x}+b \dot{\boldsymbol{x}}$, a linear combination of the CoM position $\boldsymbol{x}$ and velocity $\dot{\boldsymbol{x}}$, with the time constant $b:=\sqrt{\frac{\Delta z}{g}}$, where $\Delta z$ represents the average CoM height above the ground surface, and $g$ denotes the gravitational constant. A closely related concept, the Virtual Repellent Point (VRP) $\boldsymbol{v}$, was introduced in [4] as $\boldsymbol{v}:=\boldsymbol{x}-b^{2} \ddot{\boldsymbol{x}}$, a linear combination of the CoM position $\boldsymbol{x}$ and acceleration $\ddot{\boldsymbol{x}}$. The VRP encodes the effects of the total force (gravity and all external forces) acting on the CoM. The relation between DCM and VRP is found to be

$$
\dot{\boldsymbol{\xi}}=\frac{1}{b}(\boldsymbol{\xi}-\boldsymbol{v}) \text {. }
$$

\section{A. DCM reference trajectory generation}

In this section, we revisit the main results from [5]. For a sequence of $n$ footsteps given as foot centers $\left(\boldsymbol{p}_{i}\right)_{i=1}^{n}$, the DCM reference trajectory is generated by alternating single and double support phases with duration $T_{S S}$ and $T_{D S}$, respectively. In this work, the term step is used to describe a single support phase and the subsequent double support phase. In the following, we write the VRP and DCM reference trajectories for an arbitrary step with index $i$, using $t$ to denote the local time within each phase. First, a VRP waypoint $\boldsymbol{v}_{i}$ is placed over the foot center at a height equal to $\Delta z$

$$
\boldsymbol{v}_{i}=\boldsymbol{p}_{i}+\left(\begin{array}{lll}
0 & 0 \Delta z
\end{array}\right)^{T}
$$

During the single support phase, the reference VRP is constant

$$
\boldsymbol{v}_{i, S S}(t)=\boldsymbol{v}_{i}
$$

The DCM reference trajectory is obtained by solving (1) with $\boldsymbol{\xi}_{i, S S}\left(T_{S S}\right)=\boldsymbol{\xi}_{i, D S, 0}$ as boundary condition:

$$
\boldsymbol{\xi}_{i, S S}(t)=\left(1-\gamma_{S S}(t)\right) \boldsymbol{v}_{i}+\gamma_{S S}(t) \boldsymbol{\xi}_{i, D S, 0},
$$

where $\gamma_{S S}(t)=e^{\frac{t-T_{S S}}{b}}$, and $\boldsymbol{\xi}_{i, D S, 0}$ denotes the DCM start point of the double support phase. The DCM start point of the single support phase $\boldsymbol{\xi}_{i, S, 0}:=\boldsymbol{\xi}_{i, S S}(0)$ can be written as

$$
\boldsymbol{\xi}_{i, S S, 0}=\left(1-\gamma_{S S, 0}\right) \boldsymbol{v}_{i}+\gamma_{S S, 0} \boldsymbol{\xi}_{i, D S, 0},
$$

where $\gamma_{S S, 0}:=\gamma_{S S}(0)$.

For the double support phase, the reference VRP trajectory is designed as a linear interpolation between the current VRP $\boldsymbol{v}_{i}$ and the VRP corresponding to the next step, $\boldsymbol{v}_{i+1}$ :

$$
\boldsymbol{v}_{i, D S}(t)=\left(1-\frac{t}{T_{D S}}\right) \boldsymbol{v}_{i}+\frac{t}{T_{D S}} \boldsymbol{v}_{i+1} .
$$

A closed-form solution for the DCM reference trajectory is obtained by replacing (6) in (1) and solving the resulting differential equation. As a boundary condition, we choose $\boldsymbol{\xi}_{i, D S}\left(T_{D S}\right)=\boldsymbol{\xi}_{i+1, S S, 0}$, where $\boldsymbol{\xi}_{i+1, S S, 0}$ denotes the DCM start point of the following single support phase, and is computed by evaluating (5) for the step with index $i+1$. This approach guarantees the continuity of the complete DCM

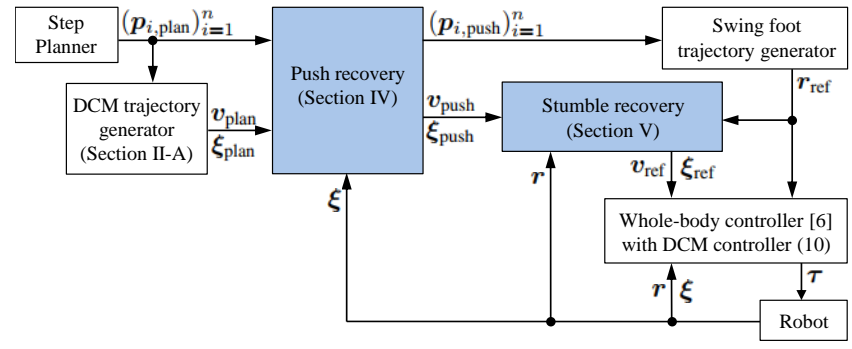

Fig. 1: Overview of the reference trajectory generator. The main contributions of this work are highlighted in blue.

trajectory (for more details, see [5]). The DCM reference trajectory for the double support phase is

$$
\boldsymbol{\xi}_{i, D S}(t)=\alpha_{D S}(t) \boldsymbol{v}_{i}+\beta_{D S}(t) \boldsymbol{v}_{i+1}+\gamma_{D S}(t) \boldsymbol{\xi}_{i+1, S S, 0},
$$

where $\alpha_{D S}(t)=1-\frac{t}{T_{D S}}-\frac{b}{T_{D S}}\left(1-e^{\frac{t-T_{D S}}{b}}\right), \beta_{D S}(t)=$ $\frac{t}{T_{D S}}-e^{\frac{t-T_{D S}}{b}}+\frac{b}{T_{D S}}\left(1-e^{\frac{t-T_{D S}}{b}}\right)$, and $\gamma_{D S}(t)=e^{\frac{t-T_{D S}}{b}}$. An important property of this formulation is that the coefficients $\alpha_{D S}(t), \beta_{D S}(t)$, and $\gamma_{D S}(t)$ are convex coefficients [17], i.e., are nonnegative and sum to 1 :

$$
\alpha_{D S}(t)+\beta_{D S}(t)+\gamma_{D S}(t)=1
$$

The DCM start point for the double support phase $\boldsymbol{\xi}_{i, D S, 0}:=\boldsymbol{\xi}_{i, D S}(0)$ can be written as:

$$
\boldsymbol{\xi}_{i, D S, 0}=\alpha_{D S, 0} \boldsymbol{v}_{i}+\beta_{D S, 0} \boldsymbol{v}_{i+1}+\gamma_{D S, 0} \boldsymbol{\xi}_{i+1, S S, 0},
$$

where $\alpha_{D S, 0}:=\alpha_{D S}(0), \beta_{D S, 0}:=\beta_{D S}(0)$, and $\gamma_{D S, 0}:=$ $\gamma_{D S}(0)$. Note that all equations in this section show linear relations between the VRP and DCM, which allows us to rewrite them later using displacements.

\section{B. DCM tracking controller}

To track the DCM reference trajectory, the following tracking control law was proposed in [4]:

$$
\boldsymbol{v}=\boldsymbol{v}_{\text {ref }}+\left(\boldsymbol{I}+b \boldsymbol{K}_{\xi}\right)\left(\boldsymbol{\xi}-\boldsymbol{\xi}_{\text {ref }}\right),
$$

where $\boldsymbol{K}_{\xi}$ is a positive diagonal matrix, $\boldsymbol{\xi}$ denotes the measured DCM, $\boldsymbol{\xi}_{\text {ref }}$ the reference DCM position, while $\boldsymbol{v}_{\text {ref }}=\boldsymbol{\xi}_{\text {ref }}-b \dot{\boldsymbol{\xi}}_{\text {ref }}$ denotes the reference VRP.

\section{OVERVIEW}

An overview of the proposed reference trajectory generator is shown in Figure 1. Based on a sequence of planned footsteps $\left(\boldsymbol{p}_{i, \text { plan }}\right)_{i=1}^{n}$ provided by an external step planner, the planned VRP and DCM trajectories, $\boldsymbol{v}_{\text {plan }}$ and $\boldsymbol{\xi}_{\text {plan }}$, are generated using the approach presented in Section II-A. In our previous work [6], these trajectories were given as reference trajectories directly to the whole-body controller. In this work, we propose an online trajectory adaptation to account for large perturbations acting on the center-ofmass (push recovery, see Section IV) and on the swing foot (stumble recovery, see Section $\mathrm{V}$ ). The result of the push recovery is an adjusted sequence of footsteps $\left(\boldsymbol{p}_{i, \text { push }}\right)_{i=1}^{n}$, used for computing the swing foot reference trajectory $\boldsymbol{r}_{\text {ref }}$ and the corresponding VRP and DCM trajectories, $\boldsymbol{v}_{\text {push }}$ and 


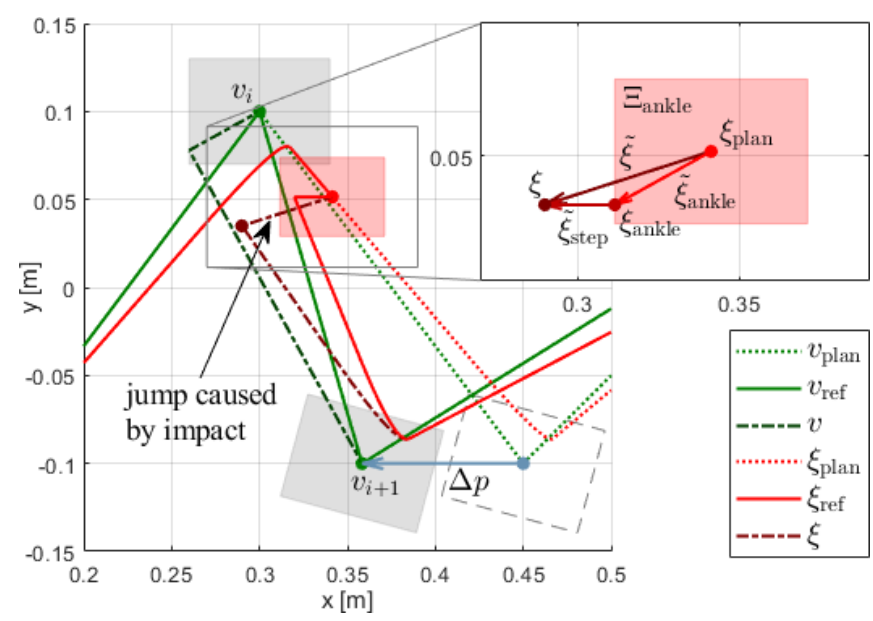

Fig. 2: Push recovery example while walking (single/double support time: $0.6 / 0.2 \mathrm{~s}$ ). The impact occurs at $t=0.27 \mathrm{~s}$ during the single support phase. The DCM error $\tilde{\xi}$ is split into two parts, $\tilde{\boldsymbol{\xi}}_{\text {ankle }}$ and $\tilde{\boldsymbol{\xi}}_{\text {step }}$, with the latter being used by the stepping strategy to compute the footstep adjustment $\Delta p$.

$\boldsymbol{\xi}_{\text {push }}$. Next, the DCM trajectory is adjusted based on the swing foot tracking error (the difference between the actual swing foot position $\boldsymbol{r}$ and the reference $\boldsymbol{r}_{\text {ref }}$ ) to create the reference VRP and DCM trajectories, $\boldsymbol{v}_{\text {ref }}$ and $\boldsymbol{\xi}_{\text {ref }}$. These are finally tracked by the DCM controller (10), which is embedded in the whole-body torque controller [6].

\section{Push Recovery}

Typically, robust push recovery is achieved by combining three strategies: ankle, stepping, and hip strategies. In our framework, the ankle strategy is implemented by the wholebody controller [6], while a hip strategy using angular momentum is part of our current research efforts. The proposed stepping strategy is implemented as an adjustment of the planned trajectories, both for the DCM and the feet. In general, it is preferable to follow the planned foot and DCM trajectories, and activate the stepping strategy only when the ankle strategy can no longer correct the DCM error.

\section{A. Stepping strategy}

In this work, we propose an analytical solution based on the DCM dynamics for implementing the stepping strategy. Let $\tilde{\xi}$ denote the difference between the measured DCM and the planned trajectory $\tilde{\boldsymbol{\xi}}(t):=\boldsymbol{\xi}(t)-\boldsymbol{\xi}_{\text {plan }}(t)$. The DCM error $\tilde{\boldsymbol{\xi}}$ can be split into two parts, $\tilde{\boldsymbol{\xi}}_{\text {ankle }}$ and $\tilde{\boldsymbol{\xi}}_{\text {step }}$ :

$$
\tilde{\boldsymbol{\xi}}_{\text {ankle }}(t)+\tilde{\boldsymbol{\xi}}_{\text {step }}(t)=\tilde{\boldsymbol{\xi}}(t),
$$

where $\tilde{\xi}_{\text {ankle }}$ represents the DCM error that can be corrected by the ankle strategy, and $\tilde{\boldsymbol{\xi}}_{\text {step }}$ represents the remaining DCM error, used by the stepping strategy to compute the next footstep adjustment $\Delta p$ (see Fig. 2). One goal of the push recovery algorithm is to follow the planned foot trajectory as close as possible, i.e. to minimize $\|\Delta p\|$. In our framework, the relation between $\tilde{\xi}_{\text {step }}$ and $\Delta p$ is linear with a scalar factor (see Section IV-C), therefore, this goal can be achieved by minimizing $\left\|\tilde{\boldsymbol{\xi}}_{\text {step }}\right\|$. Note that this linear relation is not based on heuristics, but is a direct result of the DCM dynamics (1) and the designed VRP reference trajectories (3) and (6).

The main idea of the proposed method is ilustrated geometrically in Figure 2. First, we compute the set of DCM errors $\tilde{\Xi}_{\text {ankle }}(t)$ that the ankle strategy can correct during the current step without the need for footstep adjustment (the details of the computation are given in Section IV-B). Let $\Xi_{\text {ankle }}(t)$ be the set of DCM positions for which a recovery is possible using only the ankle strategy

$$
\Xi_{\text {ankle }}=\left\{\boldsymbol{\xi}_{\text {plan }}\right\} \oplus \tilde{\Xi}_{\text {ankle }}=\left\{\boldsymbol{\xi}_{\text {plan }}+\tilde{\boldsymbol{\xi}}_{\text {ankle }} \mid \tilde{\boldsymbol{\xi}}_{\text {ankle }} \in \tilde{\Xi}_{\text {ankle }}\right\} \text {. }
$$

Projecting $\boldsymbol{\xi}$ onto $\Xi_{\text {ankle }}$ yields $\boldsymbol{\xi}_{\text {ankle }}$ that minimizes the norm $\left\|\boldsymbol{\xi}-\boldsymbol{\xi}_{\text {ankle }}\right\|$, which, as shown in Figure 2, is the same as minimizing $\left\|\tilde{\boldsymbol{\xi}}_{\text {step }}\right\|$. Note that if $\boldsymbol{\xi}$ is contained by the set $\Xi_{\text {ankle }}$ (or, equivalently, $\tilde{\xi}$ is contained by $\tilde{\Xi}_{\text {ankle }}$ ), then the stepping strategy is inactive, as $\boldsymbol{\xi}_{\text {ankle }}=\boldsymbol{\xi}$ and $\tilde{\boldsymbol{\xi}}_{\text {step }}=\mathbf{0}$.

\section{B. Set of DCM errors correctable by ankle strategy alone}

The ankle strategy corresponds to a modulation of the commanded VRP $\boldsymbol{v}$ according to (10) in the $x y$-plane, within the boundaries of the current support area. Let $\tilde{V}$ denote the set of VRP adjustments $\tilde{\boldsymbol{v}}=\boldsymbol{v}-\boldsymbol{v}_{\text {plan }}$ that remain within the foot boundaries, expressed in foot coordinates. For example, based on our choice of placing the VRP over the foot center, and given a rectangular foot shape ${ }^{1}$ of length $2 l$ and width $2 w, \tilde{V}$ can be written as $\tilde{V}=\left\{\tilde{\boldsymbol{v}}=\left(\begin{array}{lll}x & y & 0\end{array}\right)^{T} \mid-l \leqslant x \leqslant l,-w \leqslant y \leqslant w\right\}$. Let $\tilde{V}_{\text {ankle }}(t)$ denote the set of VRP adjustments that the ankle strategy can use during the current phase. In this section, we use $\tilde{V}$ and the equations derived in Section II-A to compute $\tilde{V}_{\text {ankle }}$ and $\tilde{\Xi}_{\text {ankle }}$ for single and double support phases.

1) Single support phase: The set of VRP adjustments that the ankle strategy can use during the single support phase is

$$
\tilde{V}_{\text {ankle }}=\boldsymbol{R}_{i} \otimes \tilde{V}:=\left\{\boldsymbol{R}_{i} \tilde{\boldsymbol{v}} \mid \tilde{\boldsymbol{v}} \in \tilde{V}\right\},
$$

where $\boldsymbol{R}_{i}$ denotes the rotation matrix corresponding to the yaw angle of the support foot. The corresponding set of possible commanded VRPs can be written as $V_{\text {ankle }}=\left\{\boldsymbol{v}_{i}\right\} \oplus \tilde{V}_{\text {ankle }}$.

To compute $\tilde{\Xi}_{\text {ankle }}(t)$, we start by replacing (9) in (4) and expressing the result in terms of displacements (i.e., differences between measured and planned quantities):

$$
\begin{aligned}
& \tilde{\boldsymbol{\xi}}_{i, S S}(t)=\left(1-\gamma_{S S}(t)+\gamma_{S S}(t) \alpha_{D S, 0}\right) \tilde{\boldsymbol{v}}_{i} \\
& \quad+\gamma_{S S}(t) \beta_{D S, 0} \tilde{\boldsymbol{v}}_{i+1}+\gamma_{S S}(t) \gamma_{D S, 0} \tilde{\boldsymbol{\xi}}_{i+1, S S, 0} .
\end{aligned}
$$

Now, $\tilde{\Xi}_{\text {ankle }}(t)$ is obtained by evaluating (14) for all $\tilde{\boldsymbol{v}}_{i} \in \tilde{V}_{\text {ankle }}$, and taking $\tilde{\boldsymbol{v}}_{i+1}=\mathbf{0}$ (such that no ankle strategy is required during the next step) and $\tilde{\boldsymbol{\xi}}_{i+1, S S, 0}=\mathbf{0}$ (the DCM error is completely corrected at the end of the current step):

$$
\tilde{\Xi}_{\text {ankle }}(t)=\left(1-\gamma_{S S}(t)+\gamma_{S S}(t) \alpha_{D S, 0}\right) \tilde{V}_{\text {ankle }} .
$$

\footnotetext{
${ }^{1}$ Other convex shapes of the foot can also be modeled using linear inequalities (H-representation).
} 

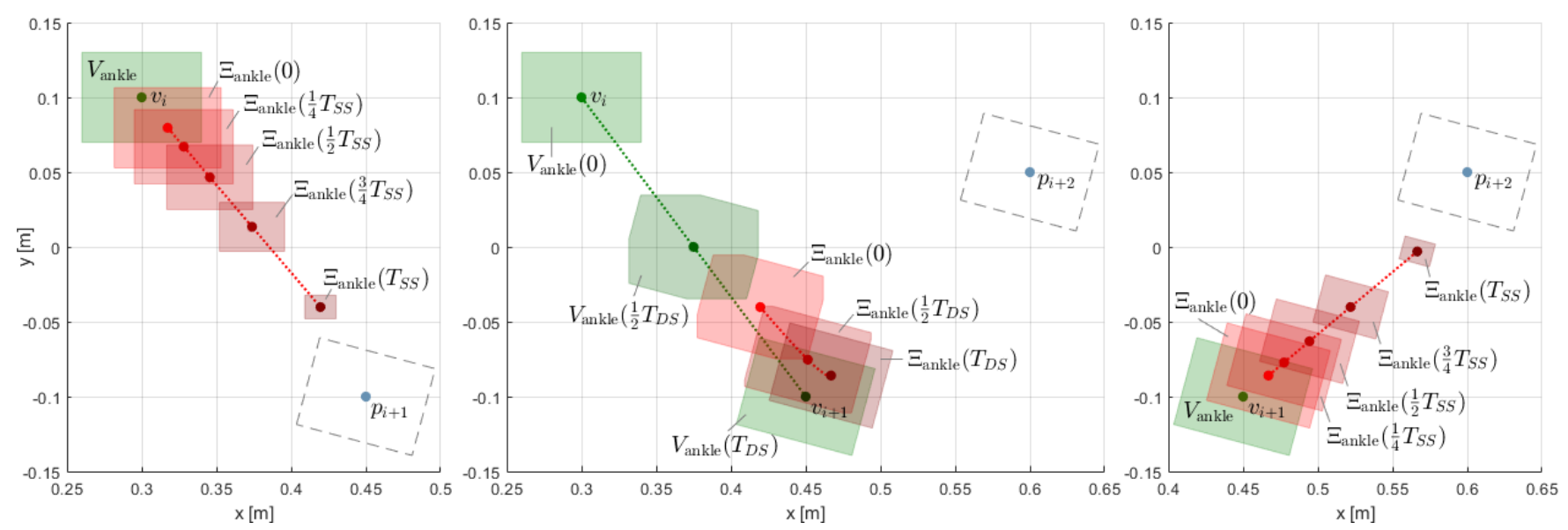

Fig. 3: Time evolution of $V_{\text {ankle }}(t)$ and $\Xi_{\text {ankle }}(t)$ during single support (left), double support (middle), and the following single support phase (right). The single and double support durations are $T_{S S}=0.6 \mathrm{~s}$ and $T_{D S}=0.2 \mathrm{~s}$, respectively.

Computing $\tilde{\Xi}_{\text {ankle }}$ using the two conditions $\left(\tilde{\boldsymbol{v}}_{i+1}=\mathbf{0}\right.$ and $\tilde{\boldsymbol{\xi}}_{i+1, S S, 0}=\mathbf{0}$ ) can also be interpreted as follows: if no further perturbations occur, any DCM error $\tilde{\xi}_{\text {ankle }} \in \tilde{\Xi}_{\text {ankle }}$ can be corrected by the ankle strategy alone until the end of the current step, using only VRP adjustments from $\tilde{V}_{\text {ankle }}$.

2) Double support phase: First, we introduce the sets $\tilde{V}_{i}=\boldsymbol{R}_{i} \otimes \tilde{V}$, and $\tilde{V}_{i+1}=\boldsymbol{R}_{i+1} \otimes \tilde{V}$, with $\boldsymbol{R}_{i}$ and $\boldsymbol{R}_{i+1}$ denoting the yaw rotation matrices of the respective feet; the corresponding VRP sets are $V_{i}=\left\{\boldsymbol{v}_{i}\right\} \oplus \tilde{V}_{i}$ and $V_{i+1}=\left\{\boldsymbol{v}_{i+1}\right\} \oplus \tilde{V}_{i+1}$.

One approach for computing the set $V_{\text {ankle }}$ of possible commanded VRPs would be to use the total support area, i.e. the convex hull of the set $V_{i} \cup V_{i+1}$. In this case, the ankle strategy could correct large disturbances that occur on the axis connecting $\boldsymbol{v}_{i}$ and $\boldsymbol{v}_{i+1}$. However, it is important to remember that during walking, the double support phase can be better described as a weight transfer phase, i.e. the weight of the robot is transfered from one leg to the other. Modulating the commanded VRP within the total support area would interfere with the planned weight transfer, potentially leading to large discontinuities in the commanded leg forces at the start of the following single support phase. Based on these considerations, the set $V_{\text {ankle }}$ is designed similarly to the VRP reference trajectory (6), as a linear interpolation between $V_{i}$ and $V_{i+1}$ :

$$
V_{\text {ankle }}(t)=\left(1-\frac{t}{T_{D S}}\right) V_{i} \oplus \frac{t}{T_{D S}} V_{i+1} .
$$

Note that the similarity between (16) and (6) allows us to compute the set $\tilde{\Xi}_{\text {ankle }}(t)$ starting from equation (7). The corresponding set of VRP adjustments can be written as

$$
\tilde{V}_{\text {ankle }}=V_{\text {ankle }} \ominus\left\{\boldsymbol{v}_{i, D S}\right\}:=\left\{\boldsymbol{v}_{\text {ankle }}-\boldsymbol{v}_{i, D S} \mid \boldsymbol{v}_{\text {ankle }} \in V_{\text {ankle }}\right\} \text {. }
$$

To compute $\tilde{\Xi}_{\text {ankle }}(t)$, we start by writing equations (5) and (9) for the single and double support phases of the $i+1$ step:

$$
\begin{aligned}
\boldsymbol{\xi}_{i+1, S S, 0} & =\left(1-\gamma_{S S, 0}\right) \boldsymbol{v}_{i+1}+\gamma_{S S, 0} \boldsymbol{\xi}_{i+1, D S, 0} \\
\boldsymbol{\xi}_{i+1, D S, 0} & =\alpha_{D S, 0} \boldsymbol{v}_{i+1}+\beta_{D S, 0} \boldsymbol{v}_{i+2}+\gamma_{D S, 0} \boldsymbol{\xi}_{i+2, S S, 0} .
\end{aligned}
$$

Combining (7) and (18), expressing the result in terms of displacements, and using the equality (8) yields

$$
\begin{aligned}
& \tilde{\boldsymbol{\xi}}_{i, D S}(t)=\alpha_{D S}(t) \tilde{\boldsymbol{v}}_{i} \\
& \quad+\left(1-\alpha_{D S}(t)-\gamma_{D S}(t) \gamma_{S S, 0}\left(1-\alpha_{D S, 0}\right)\right) \tilde{\boldsymbol{v}}_{i+1} \\
& \quad+\gamma_{D S}(t) \beta_{D S, 0} \tilde{\boldsymbol{v}}_{i+2}+\gamma_{D S}(t) \gamma_{D S, 0} \tilde{\boldsymbol{\xi}}_{i+2, S S, 0}
\end{aligned}
$$

Finally, $\tilde{\Xi}_{\text {ankle }}(t)$ is obtained by evaluating (19) for all $\tilde{\boldsymbol{v}}_{i} \in \tilde{V}_{i}$, and all $\tilde{\boldsymbol{v}}_{i+1} \in \tilde{V}_{i+1}$, while taking $\tilde{\boldsymbol{v}}_{i+2}=\mathbf{0}$ and $\tilde{\boldsymbol{\xi}}_{i+2, S S, 0}=\mathbf{0}$ (with the same interpretation as in the case of the single support phase):

$$
\begin{aligned}
& \tilde{\Xi}_{\text {ankle }}(t)=\alpha_{D S}(t) \tilde{V}_{i} \\
& \quad \oplus\left(1-\alpha_{D S}(t)-\gamma_{D S}(t) \gamma_{S S, 0}\left(1-\alpha_{D S, 0}\right)\right) \tilde{V}_{i+1} .
\end{aligned}
$$

Figure 3 shows the time evolution of $V_{\text {ankle }}(t)$ and $\Xi_{\text {ankle }}(t)$ for an example trajectory during three consecutive phases.

It can be verified that $\tilde{\Xi}_{\text {ankle }}$ is the same at the end of the double support phase and the start of the following single support phase. Evaluating (20) for $t=T_{D S}$ yields

$$
\tilde{\Xi}_{\text {ankle }}\left(T_{D S}\right)=\left(1-\gamma_{S S, 0}+\gamma_{S S, 0} \alpha_{D S, 0}\right) \tilde{V}_{i+1},
$$

where we used the identities $\alpha_{D S}\left(T_{D S}\right)=0$ and $\gamma_{D S}\left(T_{D S}\right)=1$. As $\tilde{V}_{i+1}$ is the same as $\tilde{V}_{\text {ankle }}$ during the following single support phase, the result in (21) is identical to the one obtained by evaluating (15) for $t=0$. On the other hand, $\tilde{\Xi}_{\text {ankle }}$ is discontinuous at the end of the single support phase and the start of the following double support phase (see Fig. 3, left and middle images). However, this discontinuity in the time evolution of $\tilde{\Xi}_{\text {ankle }}$ coincides with the discontinuous change of the adjustable footstep from $\boldsymbol{p}_{i+1}$ during single support, to $\boldsymbol{p}_{i+2}$ during double support.

\section{Footstep adjustment}

The stepping strategy computes a footstep adjustment $\Delta \boldsymbol{p}(t)$ based on the DCM error component $\tilde{\boldsymbol{\xi}}_{\text {step }}(t)$ that cannot be corrected by the ankle strategy alone. As a direct consequence of the DCM dynamics (1) and the designed VRP trajectories (3) and (6), the relation between the two 
quantities is linear with a scalar factor and can be expressed as

$$
\Delta \boldsymbol{p}(t)=\frac{1}{f_{\text {step }}(t)} \tilde{\boldsymbol{\xi}}_{\text {step }}(t),
$$

where $f_{\text {step }}(t)$ can be computed from the DCM trajectory equations (4) and (7).

1) Single support phase: During the single support phase, the footstep to be adjusted by the stepping strategy is $\boldsymbol{p}_{i+1}$. Due to the linearity of the relations presented so far, and the fact that only scalars were used as factors, $f_{\text {step }}$ can be obtained from the partial derivative

$$
\frac{\partial \boldsymbol{\xi}_{i, S S}(t)}{\partial \boldsymbol{p}_{i+1}}=\frac{\partial \boldsymbol{\xi}_{i, S S}(t)}{\partial \boldsymbol{v}_{i+1}} \frac{\partial \boldsymbol{v}_{i+1}}{\partial \boldsymbol{p}_{i+1}}=f_{\text {step }}(t) \boldsymbol{I} .
$$

From (2), we obtain $\frac{\partial \boldsymbol{v}_{i+1}}{\partial \boldsymbol{p}_{i+1}}=\boldsymbol{I}$. Replacing (4), (9), and (18) in (23), yields $f_{\text {step }}(t)$ for the single support phase

$$
f_{\text {step }}(t)=\gamma_{S S}(t)\left(1-\alpha_{D S, 0}\right)\left(1-\gamma_{S S, 0} \gamma_{D S, 0}\right)
$$

It can be easily verified that $0<f_{\text {step }}(t)<1, \forall t \in\left[0, T_{S S}\right]$, using the convex properties of the coefficients $\alpha_{D S}, \gamma_{D S}$, and $\gamma_{S S}$ [17].

2) Double support phase: During the double support phase, the adjustable footstep is $\boldsymbol{p}_{i+2}$, which means that $f_{\text {step }}$ can be obtained from the partial derivative

$$
\frac{\partial \boldsymbol{\xi}_{i, D S}(t)}{\partial \boldsymbol{p}_{i+2}}=\frac{\partial \boldsymbol{\xi}_{i, D S}(t)}{\partial \boldsymbol{v}_{i+2}} \frac{\partial \boldsymbol{v}_{i+2}}{\partial \boldsymbol{p}_{i+2}}=f_{\text {step }}(t) \boldsymbol{I} .
$$

Replacing (7), (18), and the equations for single and double support phases of the $i+2$ step in (25), and using $\frac{\partial \boldsymbol{v}_{i+2}}{\partial \boldsymbol{p}_{i+2}}=\boldsymbol{I}$, obtained from (2), yields $f_{\text {step }}(t)$ for the double support phase

$$
f_{\text {step }}(t)=\gamma_{D S}(t) \gamma_{S S, 0}\left(1-\alpha_{D S, 0}\right)\left(1-\gamma_{S S, 0} \gamma_{D S, 0}\right) \text {. }
$$

Again, it can be verified that $0<f_{\text {step }}(t)<1, \forall t \in\left[0, T_{D S}\right]$.

Similar to the DCM error set $\tilde{\Xi}_{\text {ankle }}$ from the previous section, the scalar factor $f_{\text {step }}(t)$ is continuous during the transition from double to single support, and discontinuous during the transition from single to double support. This can be verified by evaluating (24) and (26) at the boundaries of the local time interval. The discontinuity of $f_{\text {step }}(t)$ coincides with the discontinuous change of the adjustable footstep.

\section{Stumble ReCOVERY}

Using the same notation as in Section III, during the single support phase of the $i$-th step, the swing foot moves towards the footstep location $\boldsymbol{p}_{i+1, \mathrm{push}}$, computed by the push recovery algorithm (see Section IV). According to (2), this is the same as the $x y$-plane projection of the corresponding VRP $\boldsymbol{v}_{i+1, \text { push }}$. Let $\tilde{\boldsymbol{p}}_{i+1}$ denote the touchdown position error $\tilde{\boldsymbol{p}}_{i+1}:=\boldsymbol{p}_{i+1}-\boldsymbol{p}_{i+1, \text { push }}$, where $\boldsymbol{p}_{i+1}$ represents the actual touchdown location of the swing foot. The touchdown error may be caused by tracking errors due to model inaccuracies, by external forces acting on the swing foot, or by interaction with the ground during the touchdown approach such as when walking on compliant or uneven terrain. This error requires an adjustment of the corresponding VRP reference, otherwise the generated DCM reference trajectory during the next step may become unfeasible. A straightforward solution would be to adjust the VRP at the end of the single support phase

$$
\boldsymbol{v}_{i+1, \text { ref }}=\boldsymbol{v}_{i+1, \text { push }}+\left(\tilde{\boldsymbol{p}}_{i+1, x} \tilde{\boldsymbol{p}}_{i+1, y} 0\right)^{T} .
$$

However, this solution leads to a discontinuity in the DCM reference trajectory $\boldsymbol{\xi}_{\text {ref }}$, which translates into discontinuous commanded forces via the DCM tracking controller.

In view of these considerations, we propose a continuous DCM reference trajectory adaptation based on the instantaneous swing foot tracking error. Let $\boldsymbol{r}_{\text {ref }}$ denote the reference Cartesian position of the foot during the swing phase, $\boldsymbol{r}$ the actual position, and $\tilde{\boldsymbol{r}}$ the current tracking error $\tilde{\boldsymbol{r}}(t):=\boldsymbol{r}(t)-\boldsymbol{r}_{\text {ref }}(t)$. Using $\tilde{\boldsymbol{r}}$, the proposed continuous VRP adjustment is

$$
\boldsymbol{v}_{i+1, \text { ref }}(t)=\boldsymbol{v}_{i+1, \text { push }}+\frac{t}{T_{S S}}\left(\tilde{\boldsymbol{r}}_{x}(t) \tilde{\boldsymbol{r}}_{y}(t) 0\right)^{T} .
$$

Intuitively, the factor $\frac{t}{T_{S S}}$ can be interpreted as the probability of the touchdown error being equal to the current tracking error. This probability is small at the start of the trajectory as it can be assumed that the tracking controller reduces the error during the swing phase. However, shortly before touchdown, the probability is high that the error remains largely uncorrected. Note that evaluating (28) for $t=T_{S S}$ produces the same result as $(27)$, i.e. the final VRP adjustment is the same in both cases.

\section{Simulations AND EXPERIMENTS}

To demonstrate the robustness of the proposed methods, we have tested various scenarios in simulation using OpenHRP [18] and in experiments with TORO [19], a 27-DoFs humanoid robot developed by DLR (German Aerospace Center). Videos of the experiments and additional simulation results can be found in the multimedia attachment. The whole-body controller and the trajectory generator are implemented in Matlab/Simulink, and are executed in realtime at a rate of $1 \mathrm{kHz}$. The reference trajectory generation is computed within $45 \mu \mathrm{s}$, of which the push recovery algorithm requires $10 \mu$ s to compute a solution.

To activate the stepping strategy, a virtual foot shape of 10 $\mathrm{cm}$ length and $6 \mathrm{~cm}$ width (see Section IV-B) was used in all experiments. For comparison, TORO's foot size is $19 \mathrm{~cm} \mathrm{x}$ $9.5 \mathrm{~cm}$. This means that the stepping strategy actives early, and the DCM error component $\tilde{\boldsymbol{\xi}}_{\text {step }}$ is corrected by both the ankle and stepping strategy. To account for kinematic limitations of the robot, the maximum step size including the adjustment is limited to $\pm 20 \mathrm{~cm}$ on the $x$-axis and \pm 15 $\mathrm{cm}$ on the $y$-axis, expressed in foot coordinates. Additionally, to avoid collisions of the feet, the minimum distance between the feet on the $y$-axis is set to $18 \mathrm{~cm}$.

The first scenario (Fig. 4) is an experiment with the real robot showing two large pushes along the $x$-axis; the push magnitudes are estimated from the changes in measured linear momentum. Both pushes are strong enough to temporarily stop the robot's forward motion. Note that after the second push, at the double support phase starting at $11.1 \mathrm{~s}$, the remaining DCM error can be corrected by the 


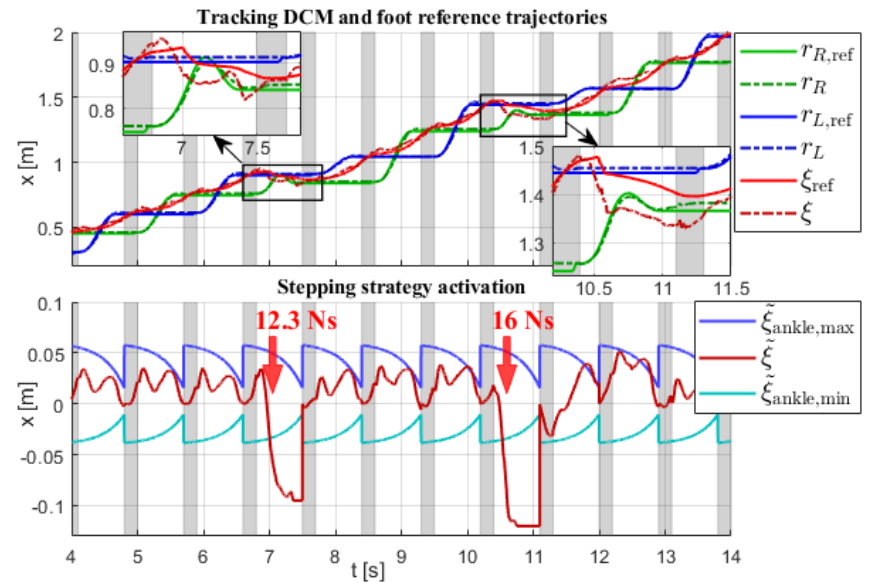

Fig. 4: Push recovery experiment during forward walking (step length: $15 \mathrm{~cm}$, single/double support time: $0.7 / 0.2 \mathrm{~s}$ ). Double support phases are shown in gray.

ankle strategy alone, without the need for further footstep adjustments.

A comparison with respect to the maximum recoverable pushes of the ankle strategy alone and the proposed combination of the ankle and stepping strategies was performed in simulation (Fig. 5). Each push occurs at $t=0.05 \mathrm{~s}$ during the single support phase on the right stance foot, and has a duration of $0.1 \mathrm{~s}$. Note that for forward pushes $\left(0^{\circ}\right.$ direction) the increase in the maximum push magnitude is limited due to the kinematic limitations of the robot. In contrast, for backward pushes $\left(180^{\circ}\right.$ direction), the stepping strategy doubles the maximum recoverable push magnitude from 18 Ns to $36 \mathrm{Ns}$, as the robot can take several steps backwards in response to the large push. The asymmetry between the maximum recoverable left $\left(90^{\circ}\right)$ and right $\left(270^{\circ}\right)$ pushes is explained by the fact that the pushes occur during the right stance phase; the left foot can be freely adjusted to the left, but its motion is limited to the right in order to avoid possible collisions with the right foot.

Finally, the DCM reference trajectory adaptation is experimentally demonstrated with the real robot (Fig. 6). The perturbations magnitudes are estimated based on the impedance forces computed by the passivity-based wholebody controller. Both disturbances act on the left foot during its swing phase, with the second disturbance leading to a touchdown error of $10 \mathrm{~cm}$ on the $x$-axis. Without the DCM reference trajectory adaptation, this large foot placement error would cause the reference VRP waypoint to be outside the actual support area during the following single support phase, leading to an unfeasible trajectory and subsequently to the robot falling. As shown in Figure 6, the DCM trajectory adaptation allows the robot to maintain its balance and continue walking.

\section{CONCLUSION AND FUTURE WORK}

In this paper, we presented a highly efficient reference trajectory generator capable of adapting online to large perturbations acting on the CoM and the feet. Our existing

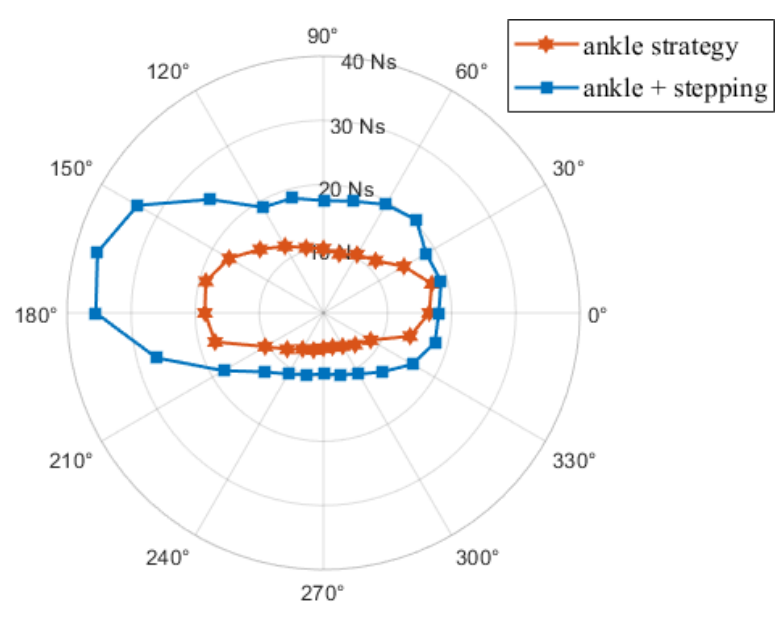

Fig. 5: Comparison of the maximum recoverable pushes while walking in simulation (step length: $15 \mathrm{~cm}$, single/double support time: 0.5/0.1 s).

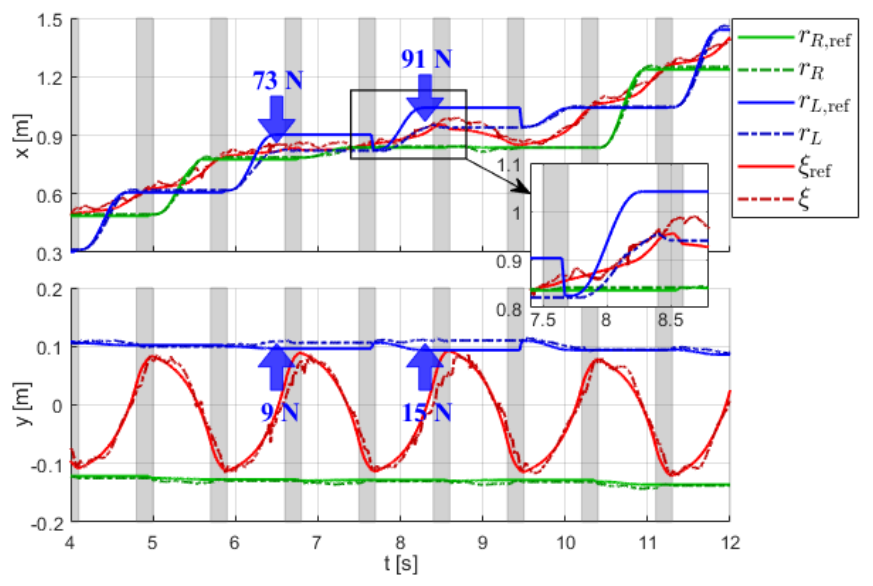

Fig. 6: DCM reference trajectory adaptation in experiment with real robot (step length: $15 \mathrm{~cm}$, single/double support time: $0.7 / 0.2 \mathrm{~s}$ ). Double support phases are shown in gray.

framework was extended with a footstep adjustment strategy for push recovery, which is active during both single and double support phases. An analytic solution was proposed for combining the ankle and the stepping strategies, including explicit double support phases. Additionally, we introduced a DCM trajectory adaptation based on foot tracking errors, making our framework capable of handling large swing foot disturbances. The robustness of the proposed method was validated with simulations and experiments on the real robot.

In our future research, we plan to extend the push recovery algorithm with step time adjustments, and expand its capabilities with a hip strategy by integrating an angular momentum task.

\section{ACKNOWLEDGEMENT}

This project has received funding from the European Research Council (ERC) under the European Union's Horizon 2020 research and innovation programme (grant agreement No. 819358). 


\section{REFERENCES}

[1] A. Kheddar, S. Caron, P. Gergondet, A. Comport, A. Tanguy, C. Ott, B. Henze, G. Mesesan, J. Englsberger, M. A. Roa et al., "Humanoid robots in aircraft manufacturing: The airbus use cases," IEEE Robotics \& Automation Magazine, vol. 26, no. 4, pp. 30-45, 2019.

[2] S. Kajita, F. Kanehiro, K. Kaneko, K. Yokoi, and H. Hirukawa, "The 3d linear inverted pendulum mode: A simple modeling for a biped walking pattern generation," in IEEE/RSJ International Conference on Intelligent Robots and Systems (IROS), vol. 1, 2001, pp. 239-246.

[3] T. Takenaka, T. Matsumoto, and T. Yoshiike, "Real time motion generation and control for biped robot-1 st report: Walking gait pattern generation," in IEEE/RSJ International Conference on Intelligent Robots and Systems (IROS), 2009.

[4] J. Englsberger, C. Ott, and A. Albu-Schäffer, "Three-dimensional bipedal walking control based on divergent component of motion," IEEE Transactions on Robotics, vol. 31, no. 2, pp. 355-368, 2015.

[5] J. Englsberger, G. Mesesan, and C. Ott, "Smooth trajectory generation and push-recovery based on divergent component of motion," in IEEE/RSJ International Conference on Intelligent Robots and Systems (IROS), 2017, pp. 4560-4567.

[6] G. Mesesan, J. Englsberger, G. Garofalo, C. Ott, and A. Albu-Schäffer, "Dynamic walking on compliant and uneven terrain using DCM and passivity-based whole-body control," in IEEE-RAS International Conference on Humanoid Robots, 2019, pp. 25-32.

[7] B. E. Maki and W. E. Mcllroy, "The role of limb movements in maintaining upright stance: the "change-in-support" strategy," Physical therapy, vol. 77, no. 5, pp. 488-507, 1997.

[8] A. Herdt, H. Diedam, P.-B. Wieber, D. Dimitrov, K. Mombaur, and M. Diehl, "Online walking motion generation with automatic footstep placement," Advanced Robotics, vol. 24, no. 5-6, pp. 719-737, 2010.

[9] Z. Aftab, T. Robert, and P.-B. Wieber, "Ankle, hip and stepping strategies for humanoid balance recovery with a single model predictive control scheme," in IEEE-RAS International Conference on Humanoid Robots, 2012, pp. 159-164.

[10] M. Shafiee-Ashtiani, A. Yousefi-Koma, and M. Shariat-Panahi, "Robust bipedal locomotion control based on model predictive control and divergent component of motion," in IEEE International Conference on Robotics and Automation (ICRA), 2017, pp. 3505-3510.

[11] J. Urata, K. Nshiwaki, Y. Nakanishi, K. Okada, S. Kagami, and M. Inaba, "Online walking pattern generation for push recovery and minimum delay to commanded change of direction and speed," in IEEE/RSJ International Conference on Intelligent Robots and Systems (IROS), 2012, pp. 3411-3416.

[12] S. Feng, X. Xinjilefu, C. G. Atkeson, and J. Kim, "Robust dynamic walking using online foot step optimization," in IEEE/RSJ International Conference on Intelligent Robots and Systems (IROS), 2016, pp. 5373-5378.

[13] T. Kamioka, H. Kaneko, T. Takenaka, and T. Yoshiike, "Simultaneous optimization of zmp and footsteps based on the analytical solution of divergent component of motion," in IEEE International Conference on Robotics and Automation (ICRA), 2018, pp. 1763-1770.

[14] R. J. Griffin, G. Wiedebach, S. Bertrand, A. Leonessa, and J. Pratt, "Walking stabilization using step timing and location adjustment on the humanoid robot, atlas," in IEEE/RSJ International Conference on Intelligent Robots and Systems (IROS), 2017, pp. 667-673.

[15] M. Shafiee, G. Romualdi, S. Dafarra, F. J. A. Chavez, and D. Pucci, "Online dcm trajectory generation for push recovery of torquecontrolled humanoid robots," in IEEE-RAS International Conference on Humanoid Robots, 2019.

[16] H. Jeong, I. Lee, J. Oh, K. K. Lee, and J.-H. Oh, "A robust walking controller based on online optimization of ankle, hip, and stepping strategies," IEEE Transactions on Robotics, vol. 35, no. 6, pp. 13671386, 2019.

[17] G. Mesesan, J. Englsberger, C. Ott, and A. Albu-Schäffer, "Convex properties of center-of-mass trajectories for locomotion based on divergent component of motion," IEEE Robotics and Automation Letters, vol. 3, no. 4, pp. 3449-3456, 2018.

[18] F. Kanehiro, H. Hirukawa, and S. Kajita, "Openhrp: Open architecture humanoid robotics platform," The International Journal of Robotics Research, vol. 23, no. 2, pp. 155-165, 2004.

[19] J. Englsberger, A. Werner, C. Ott, B. Henze, M. A. Roa, G. Garofalo, R. Burger, A. Beyer, O. Eiberger, K. Schmid, and A. Albu-Schäffer, "Overview of the torque-controlled humanoid robot TORO," in IEEERAS International Conference on Humanoid Robots, 2014, pp. 916923. 